\title{
Enhancement of cross-polarized beam components at a metamaterial surface
}

\author{
W. Szabelak • W. Nasalski
}

Received: 29 June 2010 / Revised version: 10 November 2010 / Published online: 18 December 2010

(c) The Author(s) 2010. This article is published with open access at Springerlink.com

\begin{abstract}
Beam-interface interactions under incidence of a TE polarized elegant Hermite-Gaussian beam are considered in order to obtain efficient excitation of higher-order beam modes at a homogeneous, isotropic metamaterial surface. It is shown by numerical simulations that two transmitted TE and TM polarization beam components are enhanced more efficiently at a metamaterial surface than in the case of a dielectric interface. The mixed Poynting vector contribution to the incident energy flux is evaluated to explain this enhancement. The numerical results obtained are verified against energy balance at the interface.
\end{abstract}

\section{Introduction}

Since the first Veselago's publication on artificial materials characterized by negative permittivity and negative permeability [1], many reports on theoretical and experimental aspects on metamaterials have been published. A comprehensive list of papers devoted to this topic can be found, for example, in review articles [2-4]. Due to their specific features the metamaterials offer many possibilities in designing new photonic structures. A lot of theoretical and numerical work has been done to explore properties of metamaterials suitable for specific applications. In this report, the problem of beam incidence upon an isotropic interface is considered, in the context of excitation of higher-order beam modes at such planar structures [5]. The effectiveness of this excitation, although usually rather small, could be increased by making

\section{W. Szabelak (凶) · W. Nasalski}

Institute of Fundamental Technological Research, Polish Academy of Sciences, 5B Pawińskiego St., 02-106 Warsaw, Poland

e-mail:wszab@ippt.gov.pl the beam width smaller even beyond the paraxial limit or by replacing normal incidence of the beam by its critical incidence [6]. Note that this excitation process critically depends on the incidence angle [7] and its efficiency can be further increased by using anisotropic, instead of isotropic, materials. However, it seems that, in certain circumstances, application of metamaterial isotropic surfaces may also appear suitable for the same purpose. In this report we will verify theoretically and numerically such a possibility.

The paper is organized as follows. In the next section the problem of beam transmission is specified. Elegant Hermite-Gaussian (EHG) beams [6], selected as suitable biorthogonal and complete basis in our numerical simulations, together with specification of metamaterial medium parameters, are briefly described. In Sect. 3 the enhancement of the beam amplitude during transmission through the metamaterial-air interface is presented. The beam is incident obliquely on the surface from the metamaterial side. The results are explained in terms of mixed Poynting vector theory [8-10]. The dependence of the transmitted beam amplitude on the incident beam width is also discussed. The main results are summarized in Sect. 4.

\section{Specification of the problem}

It is known that the 3D beam impinged upon the interface exhibits the cross-polarization coupling (XPC) caused by azimuthal dependence of the beam spectrum [5]. For an incident beam of the specified (TE or TM) polarization, the refracted portion of the field consists of two polarization (TE and TM) beam components. One of the two transmitted beam components has the same polarization as the incident beam and is called the direct polarization (DP) component. The second component, excited at the interface, has 


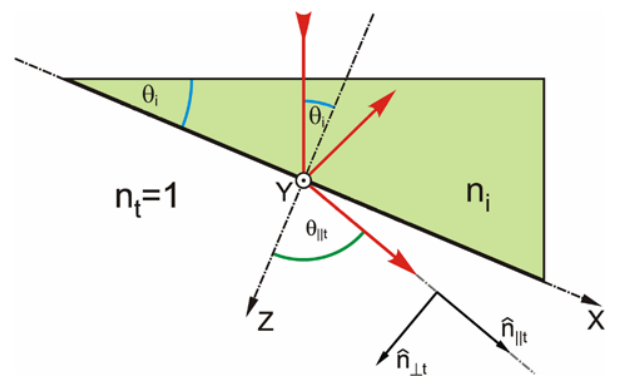

Fig. 1 Geometry of the problem. Red line points the direction of the beam propagation impinged on the surface (bold black line, $X$ axis) at the angle $\theta_{i} . n_{i}$ is the refractive index of the metamaterial or the dielectric, $\theta_{\| t}$ is the angle between real part of the wave vector of the transmitted field and normal to the interface. $\hat{n}_{\| t}$ and $\hat{n}_{\perp t}$ point the direction of phase propagation and the direction of exponentially change of the beam amplitude, respectively (see also Appendix A). The enhancement of the XPC is examined in the transmitted beam at the interface surface $(X$ axis)

the polarization orthogonal to the first one and is called the cross-polarization (CX) component. In this report a suitably chosen metamaterial-air interface is proposed to magnify the amplitude of this component, with respect to the amplitude level obtained in the case of a dielectric-air interface.

In theoretical aspects of this 3D transmission problem we follow the analysis presented in [5, 6]. The EHG beam is defined in the plane $z=$ const transverse to its propagation direction (see Fig. 1). Next, the beam is projected onto the interface plane $Z=$ const and its polarization is specified in this plane as well. It is assumed that the central spectral beam component reaches the interface at the beam waist plane defined at $z=0$. The harmonic dependence $\exp [i k z-i \omega t]$ on time $t$ and the propagation distance $z$ are assumed and suppressed. For the obliquely incident TE polarized EHG beam, each spectral plane-wave component of the transmitted beam can be written within the first-order approximation, as $[6,11]$

$\left[\begin{array}{c}\tilde{E}_{X}^{(t)} \\ \tilde{E}_{Y}^{(t)}\end{array}\right] \cong t_{s}\left[\begin{array}{l}0 \\ 1\end{array}\right] \tilde{G}_{m, n}^{(\mathrm{EH})}-\frac{i 2 t_{\mathrm{CX}}}{k_{i} w_{w} \sin \vartheta_{i}}\left[\begin{array}{l}1 \\ 0\end{array}\right] \tilde{G}_{m, n+1}^{(\mathrm{EH})}$,

where the scalar EHG beams $G_{m, n}^{(\mathrm{EH})}$ are defined in the spectral domain by their two-dimensional Fourier transforms:

$\tilde{G}_{m, n}^{(\mathrm{EH})}\left(k_{X}, k_{Y}, Z\right)=\left(i w_{w}\right)^{m+n} k_{X}^{m} k_{Y}^{n} \tilde{G}\left(k_{X}^{m}, k_{Y}^{n}, Z\right)$,

$\tilde{G}\left(k_{X}, k_{Y}, Z\right)=2 \pi \exp \left[-\frac{1}{2}\left(k_{X}^{2}+k_{Y}^{2}\right) v^{2}\right]$.

The left side of (1) represents a vector composed of two spectral components of the transmitted beam: the upper one represents the TM, CX polarization component, the lower one represents the TE, DP polarization component. The standard Fresnel and the XPC transmission coefficients are $t_{s}$ and $t_{\mathrm{CX}}=\left(\eta t_{p}-t_{s}\right) / 2$, respectively, $k_{i} w_{w}$ is the dimensionless, normalized by the wave number $1 / k_{i}$, beam half-width at the waist, $\vartheta_{i}$ is the polar incident angle of the beam spectral component. Equation (2) is a spectral representation of the incident elegant Hermite-Gaussian beam of the indices $m, n$ specified in the two transverse to $Z$ directions $[5,6]$.

One way to enhance the amplitude of the transmitted CX component is to manipulate the magnitude of the $t_{\mathrm{CX}}$ coefficient. It can be accomplished by specification of the parameters of the metamaterial medium, in which the beam is traveling, or from which is reflected. We chose the single negative metamaterial described in [12]. The maximally efficient excitation of the CX component can be achieved for the beam of TE polarization traveling from the metamaterial to air. In this case both transmitted components experience the highest magnification with relation to the dielectric interface case. The geometry of the problem is plotted in Fig. 1.

Individual elements of metamaterials are typically much smaller in size in relation to the radiation wavelength. Therefore, the metamaterials can be considered as homogeneous and their electromagnetic properties can be characterized by macroscopic parameters such as effective permittivity $\varepsilon$ and permeability $\mu$. The refractive index $n$ is then calculated from values of $\varepsilon$ and $\mu$. We consider the cut wires structure at the left border of the frequency band gap, where $n$ is not purely imaginary. It exhibits resonant dependence of the effective permittivity [12]:

$\varepsilon(v)=1-\frac{F v^{2}}{v^{2}-v_{0}^{2}+i \gamma v}$

where $\gamma$ is the damping factor and $F$ is the fraction of the metamaterial volume of the metallic components, $v$ and $v_{0}$ are the frequency and the resonance frequency, respectively. Then, the refractive index $n(v)=\sqrt{\varepsilon(v) \mu(v)}$ yields the frequency dependence of $\mu$. In the frequency interval considered the imaginary parts of $\varepsilon$ and $\mu$ possess opposite signs. That entails small transmission losses of the beam traveling in the structure. For different frequencies of the beam the medium possesses different complex values of $\varepsilon$ and $\mu$. The effective parameters used in this paper are showed in Table 1 [12]. They correspond to the situation when the frequency of the incident field is set close to the resonance, at the midpoint and far from the resonance, near point where real part of $n$ tends to zero.

To illustrate the enhancement of both transmitted components we simulate the case of incidence of the TE polarized $\tilde{G}_{1,1}^{(\mathrm{EH})}$ beam at the metamaterial surface. Three different sets of effective parameters of the metamaterial are chosen (see Table 1) where various angles of the incident beam were examined, first to optimize the excitation of the CX component. 
Table 1 Complex values of permittivity, permeability and the refractive index of the single negative material taken for numerical simulations. The resonant frequency is $\nu_{0} \approx 13.35 \mathrm{GHz}[12]$

\begin{tabular}{lllllrrr}
\hline $\begin{array}{l}\text { Relation to the } \\
\text { resonance }\end{array}$ & $v[\mathrm{GHz}]$ & $\varepsilon^{\prime}$ & $\varepsilon^{\prime \prime}$ & $\mu^{\prime}$ & $\mu^{\prime \prime}$ & $n^{\prime}$ \\
\hline close & 13.7 & -3.23 & 19.94 & 0.14 & -0.59 & 3.42 \\
mid & 15.3 & -3.14 & 5.66 & 0.75 & -1.25 & 2.66 \\
far & 16.7 & -3.77 & 3.50 & 1.90 & -1.71 & 2.45 \\
\hline
\end{tabular}

Fig. 2 Magnitudes of the spectral transmission coefficients (first row) and the corresponding modified transmittances (second row). First three lines are for the metamaterial interface for three various frequencies of the incident field: close to the resonance, at the midpoint and far from the resonance, see Table 1 . The fourth line is for the standard dielectric interface specified by the critical angle equal $45^{\circ}$; (a) the magnitude of the Fresnel coefficient $t_{s}$, (b) of the cross-polarization coefficient $t_{\mathrm{CX}}$. The modified transmittance (c) for the DP spectral component, (d) for the CX spectral component. Modified transmittances are defined in (7). Inset is the vertical zoom, the range equals $0-0.006$
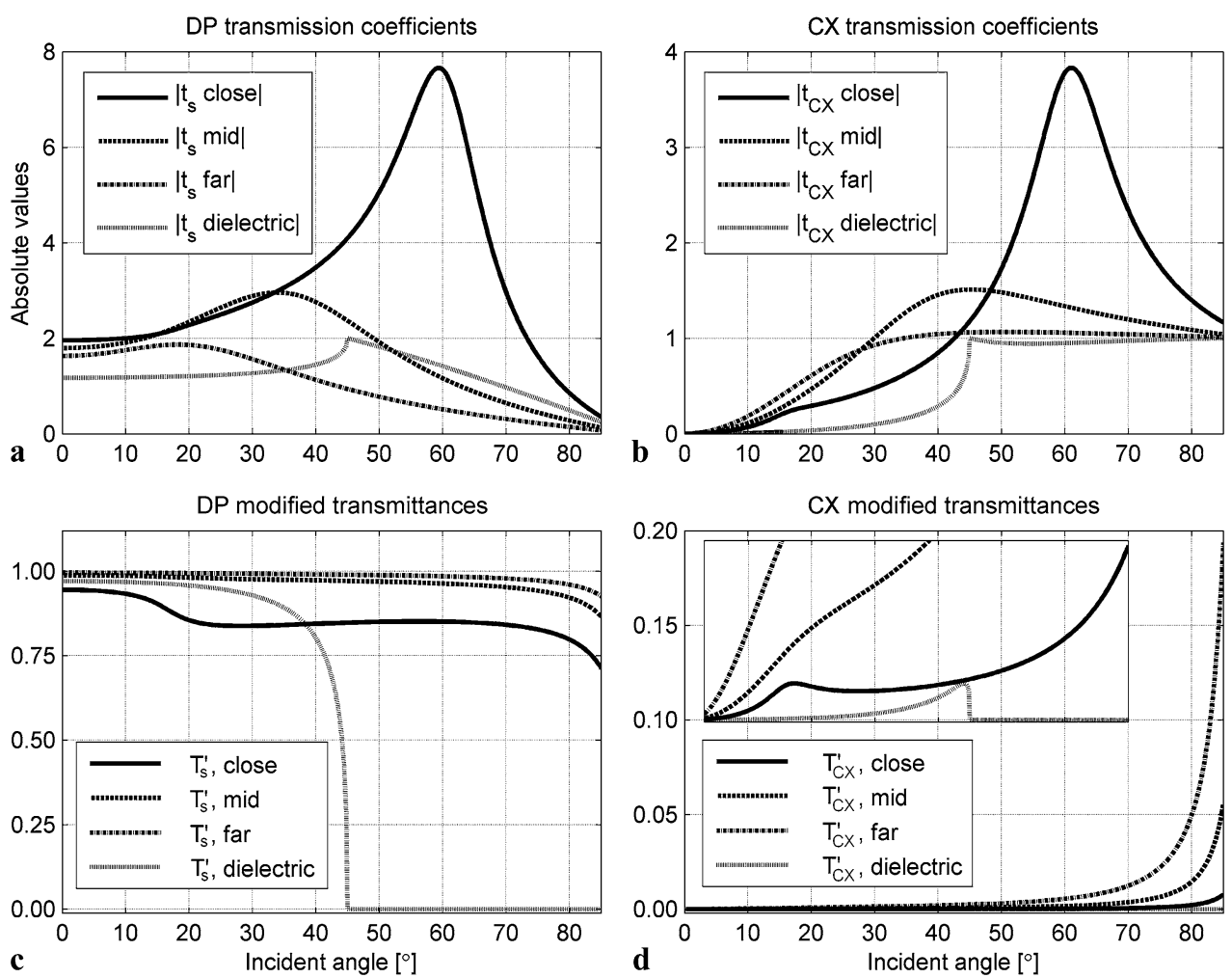

\section{Enhanced transmission through the metamaterial surface}

In the paraxial range of the beam width, the amplitudes of the transmitted (TE and TM) beam components are determined closely by magnitudes of the standard Fresnel and the XPC coefficients. Here, the DP beam component is of TE polarization and the CX beam component is of TM polarization. Magnitudes of the Fresnel and the XPC coefficients are plotted in Figs. 2(a) and (b), respectively, as functions of the plane-wave incidence angle, for the specified above metamaterial interfaces (see Table 1). An additional plot for a standard dielectric interface is also presented. Corresponding modified transmittances (specified below, see (7) and Appendix A) for the selected spectral component of the DP and the CX fields are plotted in Figs. 2(c) and (d), respectively.

The maximal amplitude of transmitted field coincides almost exactly with the maximal magnitude of the spectral coefficient for the central plane wave in the spectral decomposition of this field. Therefore, in order to examine maximal amplitudes of the transmitted field components, one can concentrate on the spectral coefficients $t_{s}$ and $t_{\mathrm{CX}}$ for the central plane waves. The maximal magnitude, greater than 7 , of the DP transmission coefficient, Fig. 2(a), is reached for the incident angle within the vicinity of $60^{\circ}$, for metamaterial when we are close to the resonance. The CX spectral coefficient reaches its maximum in the same range of incident angles. The magnitude achieved is more than three times greater than in the case of the isotropic dielectric interface. Frequency close to the resonance is not the best choice for whole range of incident angles. For less oblique incidences, the highest maximal amplitudes of the excited field are achieved for higher frequencies, i.e. at the midpoint and far from the resonance.

The greater than two amplitude of transmitted planewave component may be considered as contradictory. Note, however, that the complex Fresnel refraction coefficient 

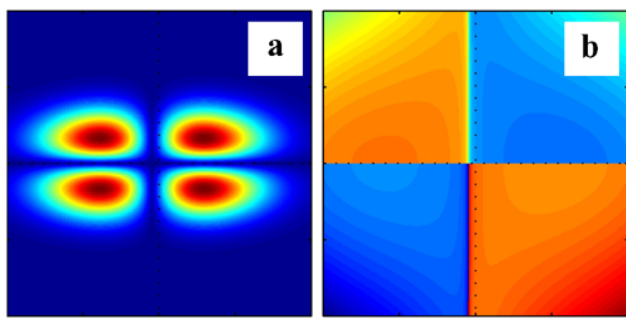

Fig. 3 Transmitted beam distributions at the metamaterial-air interface for oblique incidence of a $G_{1,1}^{(\mathrm{EH})}$ beam. The frequency of the incident field is close to the resonance. The figure shows the distribution of (a) the amplitude and (b) the phase of the DP component and of (c) the

yields an additional phase shift to the transmitted wave. The complex reflection coefficient also gives another phase shift. For the metamaterial-air interfaces, by virtue of the boundary conditions, these shifts lead to higher than two amplitudes of transmitted and reflected plane waves. To verify these amplitudes the radiant flux balance relations may be applied. The absorbing-nonabsorbing interfaces were examined more than 30 years ago in a series of papers published by Fragstein and his co-workers (see e.g. [8, 9]). A summary of those considerations, together with balance relations for nonmagnetic boundaries, expressed by terms of the Fresnel coefficients, can be found in paper [10].

For the plane waves the radiant flux balance is the real part of the conservation equation of the normal components of the complex Poynting vector (see Appendix A):

$S_{i}^{\perp}+S_{m}^{\perp}=S_{r}^{\perp}+S_{t}^{\perp}$

Here $S_{i}^{\perp}$ is the incident, $S_{r}^{\perp}$ the reflected, $S_{m}^{\perp}$ the mixed and $S_{t}^{\perp}$ the transmitted normal components of the complex Poynting vector. The real part of (5) takes the form

$\Phi_{i}^{\perp}+\Phi_{m}^{\perp}=\Phi_{r}^{\perp}+\Phi_{t}^{\perp}$.

The terms $\Phi_{i}^{\perp}=\operatorname{Re} S_{i}^{\perp}$ represents the radiant flux that arrives on the unit area of the interface, $\Phi_{r}^{\perp}=\operatorname{Re} S_{r}^{\perp}$ and $\Phi_{t}^{\perp}=\operatorname{Re} S_{t}^{\perp}$ are the parts of the flux reflected and refracted from this area, respectively, and $\Phi_{m}^{\perp}=\operatorname{Re} S_{m}^{\perp}$ is the additional mixed flux, being the consequence of medium losses and/or presence of inhomogeneous waves. The flux $\Phi_{m}^{\perp}$ may be positive and then may cause an enhancement of the transmitted radiation [8-10]. Analytical calculations indicate that $\Phi_{m}^{\perp}$ may be even two orders of magnitude greater than the incident flux $\Phi_{i}^{\perp}$, for particular angles of incidence (see Appendix A).

The radiant flux balance (6) can be rewritten in terms of the modified transmittance $R^{\prime}$ and reflectance $T^{\prime}$ (see Appendix A):

$1=R^{\prime}+T^{\prime}$
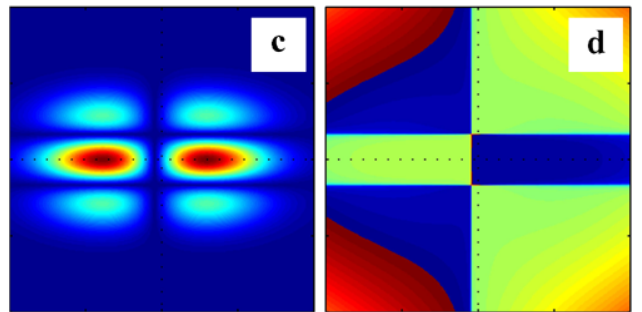

amplitude and (d) the phase of the CX component, respectively. The angle of incidence is approximately $60^{\circ}$. In the phase decompositions dark blue is for $-\pi$, dark red for $\pi$. The length of the square sides is $12 w_{w}$

where

$T^{\prime}=\Phi_{t}^{\perp} /\left(\Phi_{i}^{\perp}+\Phi_{m}^{\perp}\right)$,

$R^{\prime}=\Phi_{r}^{\perp} /\left(\Phi_{i}^{\perp}+\Phi_{m}^{\perp}\right)$.

Analytical forms of (8) and (9) for the metamaterial boundaries under consideration are presented in Appendix A ((A.5) completed by (A.4)).

Figures 2(c) and (d) show the modified transmittances $T^{\prime}$, for one spectral beam component for the two orthogonal beam polarizations, the DP and the CX, respectively. Figure 2(d) is obtained in analogical way as Fig. 2(c), but the transmission coefficients, $t_{s}$, is replaced by a function of the $t_{\mathrm{CX}}$ and the normalized spectral coordinates. The vertical zoom, shown in the inset, indicates the advantage of using the metamaterial-air interface over the dielectric-air one. Note that due to the complex refractive index of the metamaterial, the transmitted beam consists of inhomogeneous plane waves. A short explanation and the analytical form of these plane waves and corresponding radiant fluxes are shown in Appendix A.

The spatial distribution of the transmitted fields is presented in Fig. 3. It represents the most efficient (with respect to the absolute value of the CX component amplitude) case achieved at the plane of the metamaterial surface. Figures 3(a) and (b) show the amplitude and the phase of the DP component, respectively, and correspond to the maximal value of the coefficient given in Fig. 2(a). Figures 3(c) and (d) are the amplitude and the phase of the CX component, respectively, and correspond to the maximal value of the coefficient given in Fig. 2(b). The maximal amplitude in the DP polarization component is equal to 7.4 , for the CX component it is equal to 0.23 , both normalized to the maximal incident beam amplitude. In the CX component the excitation of the higher-order mode along the vertical direction is clearly visible; the index $n$ is changing from 1 to $2[6,11]$. Figure 3 also reveals a longitudinal beam displacement $[6,13]$, parallel to the interface, visible in the horizontal direction.

It is already known that, for the cross-polarization excitation at the dielectric interface, the amplitude of the excited 


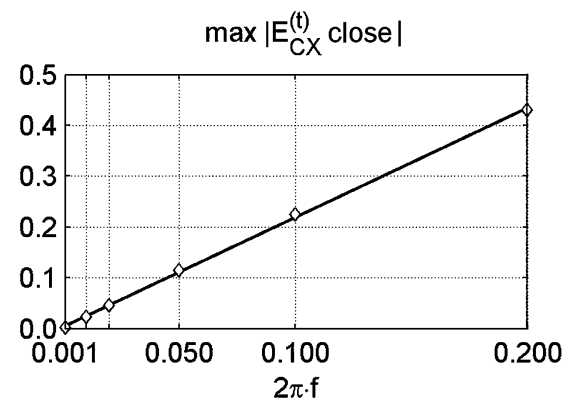

Fig. 4 Maximal amplitude of the distribution of the excited CX component with reference to the scaled angular spread of the beam $\left(2 \pi f=2 \pi / k_{i} w_{w}\right)$ at the metamaterial surface, for field frequency close to resonance. Values on the axis of the abscissa correspond to $k_{i} w_{w}=1000,100,50,20,10,5$ times $2 \pi$, respectively

component increases with decrease of the beam width [6]. We numerically verified that the same holds for the metamaterial surfaces. Figure 4 shows that the amplitude of the CX component increases linearly with increasing angular spread ( $\left.f=1 / k_{i} w_{w}\right)$ of the incident beam. Various choices of values of $k_{i} w_{w}$ from 5 to 1000 times $2 \pi$ are examined.

The linear scaling of the CX component is implied by (1). If changes of $t_{\mathrm{CX}}=t_{\mathrm{CX}}\left(\vartheta_{i}\right)$ with the incidence angle are small and can be neglected, then the transmitted beam amplitude is scaled linearly with $f$. A similar result was also derived in $[13,14]$ for the excitation of the first-order Hermite-Gaussian beams.

\section{Conclusion}

Significant enhancement of the higher-order beam-mode excitation at the isotropic metamaterial surface has been shown

TE direct polarization component

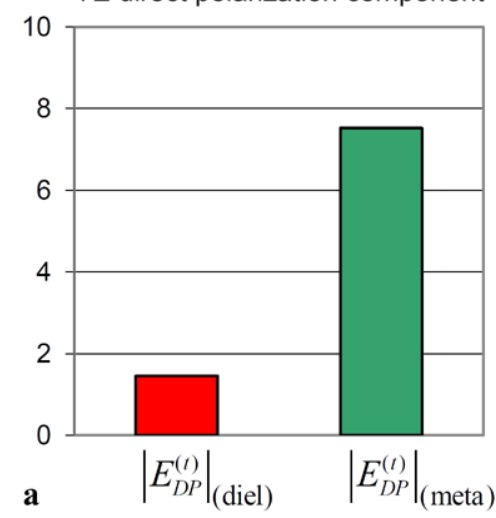

Fig. 5 Enhancement of the maximal amplitudes of the DP, (a), and the CX, (b), field orthogonal components of the transmitted beam. Red bars represent the beam amplitudes at the dielectric surface. Green bars represent the beam amplitudes at the metamaterial surface, for field frequency close to resonance. The cases (a) by numerical simulations. The results obtained were explained by the presence of the mixed flux contribution to the incident flux, which in turn enhances the radiation transmission. For the configuration discussed here both polarization components of the transmitted beam-of the direct polarization and of the opposite polarization-may be enhanced. Maximal amplitudes of the orthogonal components critically depend on the incident beam width and incidence direction. Moreover, the amplitude of the transmitted coupled (CX) beam component increases linearly with the increase of the angular spread of the incident beam. This fact confirms the complete agreement between theoretical predictions and results of our numerical simulations.

The drawing presented in Fig. 5 summarizes the result extracted from Fig. 2 (a and b) and Fig. 3, specified for the incidence angle yielding the beam transmission maximal in the beam amplitude. For this special case the maximal amplitudes of both polarization components of the beam transmitted at the metamaterial interface increase approximately by a factor of four, with respect to the case of beam transmission at the dielectric interface. For other angles of the beam incidence this factor is smaller and different for the two beam polarization components. The beam field enhancement depends, besides the medium parameters, like the effective impedance and the effective refractive index of the semi-infinite metamaterial structure, also on the (EHG) beam parameters like the beam width, the beam incidence direction and the mode indices of the incident beam. The enhancement process should depend also on the type of metamaterial medium [15] applied.

To the best of our knowledge, this is the first numerical confirmation of the possible enhancement of the crosspolarization coupling at metamaterial isotropic interfaces, we report in this paper. This coupling has been here demon-

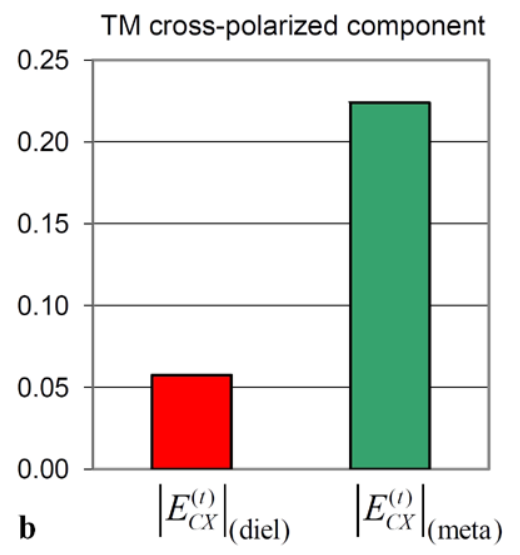

and (b) correspond to one point (angle of incidence $\theta_{i}=60^{\circ}$ ) on the axis of abscissa in Figs. 2(a) and (b). The beam amplitudes at the metamaterial interface are enhanced approximately by four times with respect to the case of the dielectric interface 
strated between the linear, TE and TM, polarization beam components, composed of elegant Hermite-Gaussian modes $[5,6]$. It seems, however, that the same coupling enhancement can be similarly accomplished in other configurations, for example between circular, left-handed and righthanded, polarization beam components, composed of elegant Laguerre-Gaussian modes [5-7]. This feature may appear particularly interesting in cases of interfaces composed of chiral metamaterial media [4].

Open Access This article is distributed under the terms of the Creative Commons Attribution Noncommercial License which permits any noncommercial use, distribution, and reproduction in any medium, provided the original author(s) and source are credited.

\section{Appendix A: Modified reflectance and transmittance for the metamaterial-air interface}

For the homogeneous TE polarized plane wave impinged on the absorbing-nonabsorbing interface, the refracted plane wave is inhomogeneous, as can be predicted from the conservation of tangential components of the wave vector at the boundary: $\hat{s} \times \vec{k}_{i}=\hat{s} \times \vec{k}_{t}$ (e.g. [10]). The inhomogeneous plane wave is, usually, described by the complex wave vector

$\vec{k}_{t}=k_{0} n_{t} \vec{n}=k_{0}\left(\cosh \beta_{t} \hat{n}_{\| t}+i \sinh \beta_{t} \hat{n}_{\perp t}\right)$.

Together with the conservation of tangential components given here as

$\left(n_{i}^{\prime}+i n_{i}^{\prime \prime}\right) \hat{s} \times \hat{k}_{i}=\cosh \beta_{t} \hat{s} \times \hat{n}_{\| t}+i \sinh \beta_{t} \hat{s} \times \hat{n}_{\perp t}$

where $n_{i}^{\prime}$ and $n_{i}^{\prime \prime}$ are the real and the imaginary parts of the refractive index of the metamaterial, respectively, $\hat{s}$ is the unitary vector normal to the interface, $\vec{k}$ is the wave vector, $\hat{n}_{\| t}$ and $\hat{n}_{\perp t}$ are the orthogonal unitary vectors connected with the complex wave vector of the inhomogeneous plane wave, and $\beta_{t}$ is a real parameter, nonzero for inhomogeneous plane waves. All these vectors are shown in Fig. 6 . In the case discussed in this paper, $\hat{n}_{\| t}$ points in the direction of phase propagation and $\hat{n}_{\perp t}$ points, if $\beta_{t}<0$, in the direction of exponential growth of the beam amplitude.

For the metamaterial-air interfaces (A.2) possesses two solutions. The first is $\beta_{t}<0$ for $0<\theta_{\| t}<\pi / 2$ and the second is $\beta_{t}>0$ for $\pi / 2<\theta_{\| t}<\pi$. The latter describes the plane wave of which the normal component of the Poynting vector is antiparallel to $Z$ direction. This solution is not covered by the radiant flux balance (6).

For absorbing-nonabsorbing boundaries, the radiant flux balance, written as the normal components of the real parts of the complex Poynting vector, $\vec{S}(\vec{r})=\left(\vec{E}(\vec{r}) \times \vec{H}^{*}(\vec{r})\right) / 2$, on two sides of the interface, has the mixed term $\vec{S}_{m}^{\perp}(\vec{r})=$ $\hat{s} \cdot\left(\vec{E}_{i}(\vec{r}) \times \vec{H}_{r}^{*}(\vec{r})+\vec{E}_{r}(\vec{r}) \times \vec{H}_{i}^{*}(\vec{r})\right) / 2$, which is nonzero

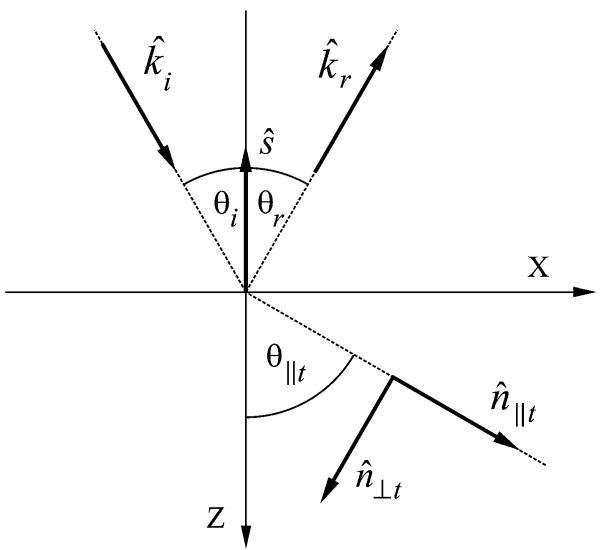

Fig. 6 Coordinate system with plotted unitary vectors and angles

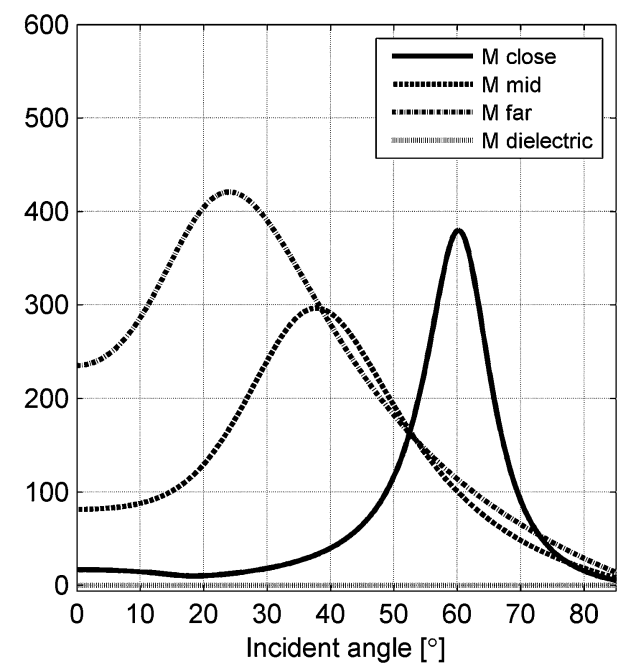

Fig. 7 Ratio $(M)$ of the mixed radiant flux to the incident flux for three metamaterial surfaces (the frequency of the incident field is close to the resonance, at the midpoint and far from the resonance) and the dielectric one, the critical angle equals $45^{\circ} . M$ depends on the imaginary part of the Fresnel reflection coefficient and the argument of the metamaterial impedance

if the imaginary part of Fresnel reflection coefficient is also nonzero [10].

The radiant flux balance, (6), can be rewritten in terms of flux coefficients, i.e.: reflection $R$, transmission $T$ and additional mixed flux coefficient $M$. By dividing (6) by $\Phi_{i}^{\perp}$ one obtains [10]

$1+M=R+T$

with

$R=\Phi_{r}^{\perp} / \Phi_{i}^{\perp}=\left|r_{s}\right|^{2}$,

$M=\Phi_{m}^{\perp} / \Phi_{i}^{\perp}=2 \operatorname{Im} r_{s} \cdot \tan \phi_{Z i^{*}}$,

$T=\Phi_{t}^{\perp} / \Phi_{i}^{\perp}=\left|Z_{i}\right|\left|t_{s}\right|^{2} \frac{\cosh \beta_{t} \cos \theta_{\| t}}{\cos \phi_{Z i^{*}} \cos \theta_{i}}$ 
where $Z_{i}=\sqrt{\mu_{i} / \varepsilon_{i}}$ is the complex impedance of the metamaterial and $\phi_{Z i *}$ is the argument of its conjugate, $Z_{i}^{*}=\left|Z_{i}\right| \exp \left[i \phi_{Z i^{*}}\right]$. For the metamaterials considered, the greater the frequency of the incident field (farther from the resonance), $\phi_{Z i} i^{*}$ is closer to $\pi / 2$. The modified reflectance and transmittance, (7), can be obtained from (A.3). Dividing by its left side gives

$R^{\prime}=R /(1+M)$,

$T^{\prime}=T /(1+M)$,

$R^{\prime}$ and $T^{\prime}$ became the standard reflectance and transmittance for nonabsorbing interfaces, respectively, for which the mixed flux coefficient, $M$, is zero. Figure 7 shows the mixed flux coefficient $M$ for medium boundaries considered in this paper.

It appears that in the case of the metamaterial-air interfaces the mixed flux becomes two orders of magnitude greater than the incident flux. To the best of the authors' knowledge this unique feature of the isotropic dissipative boundaries has not been reported yet.

\section{References}

1. V.G. Veselago, Sov. Phys. Usp. 10, 509 (1968)

2. D.R. Smith, J.B. Pendry, M.C.K. Wiltshire, Science 305, 788 (2004)

3. G. Goussetis, A.P. Feresidis, A.R. Harvey, J. Mod. Opt. 57, 1 (2010)

4. B. Wang, J. Zhou, T. Koschny, M. Kafesaki, C.M. Soukoulis, J. Opt. A, Pure Appl. Opt. 11, 114003 (2009)

5. W. Nasalski, Phys. Rev. E 74, 056613 (2006)

6. W. Nasalski, Bull. Pol. Acad. Tech. 58, 141 (2010)

7. W. Szabelak, W. Nasalski, Bull. Pol. Acad. Tech. 57, 181 (2009)

8. C. von Fragstein, F.R. Kessler, Optik 66, 9 (1983)

9. C. von Fragstein, Brauns. Wiss. Ges. 39, 25 (1987)

10. M.A. Dupertuis, B. Acklin, M. Proctor, J. Opt. Soc. Am. A 11, 1167 (1994)

11. W. Nasalski, Y. Pagani, J. Opt. A, Pure Appl. Opt. 8, 21 (2006)

12. T. Koschny, P. Markos, D.R. Smith, C.M. Soukoulis, Phys. Rev. E 68, 065602 (2003)

13. W. Nasalski, Opt. Commun. 197, 217 (2001)

14. A. Kohazi-Kis, Opt. Commun. 253, 28 (2005)

15. T. Koschny, M. Kafesaki, E.N. Economou, C.M. Soukoulis, Phys. Rev. Lett. 93, 107402 (2004) 